\title{
Machine and Human Observable Differences in Groups' Collaborative Problem-solving Behaviours
}

\author{
Mutlu Cukurova ${ }^{1}$, Rose Luckin $^{1}$, Manolis Mavrikis $^{1}$, Eva Millán ${ }^{2}$ \\ 1. UCL Knowledge Lab, University College London, United Kingdom. \\ 2. University of Málaga, Spain
}

\begin{abstract}
This paper contributes to our understanding of how to design learning analytics to capture and analyse collaborative problem-solving (CPS) in practice-based learning activities. Most research in learning analytics focuses on student interaction in digital learning environments, yet still most learning and teaching in schools occurs in physical environments. Investigation of student interaction in physical environments can be used to generate observable differences among students, which can then be used in the design and implementation of Learning Analytics. Here, we present several original methods for identifying such differences in groups CPS behaviours. Our data set is based on human observation, hand position (fiducial marker) and heads direction (face recognition) data from eighteen students working in six groups of three. The results show that the high competent CPS groups spend an equal distribution of time on their problem-solving and collaboration stages. Whereas, the low competent CPS groups spend most of their time in identifying knowledge and skill deficiencies only. Moreover, as machine observable data shows, high competent CPS groups present symmetrical contributions to the physical tasks and present high synchrony and individual accountability values. The findings have significant implications on the design and implementation of future learning analytics systems.
\end{abstract}

Keywords: Collaborative learning; problem-solving; learning analytics

\section{Introduction}

Open-ended, collaborative, practical learning activities are an essential part of STEM education and are employed as part of many common teaching approaches, including problem-based learning, inquiry-based learning, project-based learning, and practice-based learning. Such constructivist teaching approaches have potential to help foster the 21st century learning skills we require of young people across subject domains [1]. However, existing evidence on the effectiveness of these methods to satisfy common learning outcomes is rare [2, 3]. As argued by Blikstein and Worsley [4], one reason for this is that evaluation in this context is notoriously laborious and requires measurement methods that the current standardized testing strategies and psychometrics cannot provide. 
On the other hand, multimodal learning analytics (MMLA) research can yield novel methods that can generate distinctive information about what happens when students are engaged in practice-based learning activities [4]. This information can be used to inform student models, which allow to automate the support and continuous evaluation of student skills [5]. In this paper, we focus on students collaborative problem solving (CPS) ability. CPS is one of the fundamental teaching and learning strategies involved in constructivist pedagogies, such as practice-based learning. We present an empirical study through which we explored CPS behaviours in six groups of three students (aged 11-12 years) while they were working on a practice-based activity. The main goal of this study is to investigate observable differences between groups of students during CPS (both human observable and machine observable). These differences can be used to provide support for behaviours that lead to effective CPS and help automate the identification of patterns of effective CPS behaviours. In this vein, there are already some research efforts to automate the discovery of patterns of interaction that can be associated with different collaboration strategies, which can lead to more effective collaboration $[5,6]$. However, overwhelming majority of these approaches collect data from students interaction in digital learning environments. Differently in our approach, we focus on data from face-to-face learning environments.

\section{What is collaborative problem-solving?}

It is important that we make clear what we mean by the term CPS, because, as learning analytics developers, we rely on clear definitions of complex terms to drive the analysis of our data. The research questions are themselves shaped by theoretical understanding, which enables researchers to make sense of their data [8]. Drawing from these considerations, we initially start by the OECD's definition. Collaborative problem-solving competency is the capacity of an individual to effectively engage in a process whereby two or more agents attempt to solve a problem by sharing the understanding and effort required to come to a solution and pooling their knowledge, skills and efforts to reach that solution [7]. However, the OECD approach is not complete in its reflection of CPS. It should be noted that the OECD approach was developed for assessment purposes, which results in a couple of limitations. First, the process of CPS is only considered from an individual capacity perspective. This makes sense from the OECDs perspective since PISA assessments are done at the individual level. However, CPS is a multilevel process and needs to be considered from different perspectives, which must reflect the needs of individuals, groups and communities and these different perspectives should be taken into account in the design and investigation of CPS processes [9]. Second, the OECDs approach does not include some components of problem-solving, which are considered as important when CPS is considered as a tuition approach [11]. For example, it does not take into account the element of identifying each participants knowledge deficiency [10]. In this research study, we, therefore, use a theoretical framework that is based on PISAs 
exhaustive work on CPS, combined with research that has considered CPS as a tuition process.

\section{$3 \quad$ Experiment and methodology}

\section{$3.1 \quad$ Participants}

The participants were eighteen secondary school students in the first year of their secondary education (aged 11-12 years) from a girls-only secondary school in the UK. All students were recruited from a computer science class. We obtained written consent from both students and their parents/guardians in line with our institutions ethics procedures.

\subsection{Learning Activity}

The activity was conducted as part of students computer science school curriculum activity with physical computing kits. However, due to the practical issues of the transport of the learning analytics system, students and the teachers were invited to the leading author's institute to undertake the activity. Students were set the task of building a working prototype of an interactive toy using an Arduino-based physical computing kit, called TALKOO, that was created as part of an EU-funded project (www.pelars.eu). The TALKOO kit comprises hardware modules, a visual IDE and prototyping material. Sensor and actuator modules are pluggable and do not require soldering, and no prior knowledge of electronics is needed. The students were also provided with craft materials (coloured paper, paper cups, wooden sticks, glitter, glue, etc.) with which to create their working prototypes in combination with the physical computing kit.

\subsection{Sessions}

The session lasted about four hours and involved:

1. A refresher session, during which students worked through predefined activities that exemplified the functions of TALKOO components and logic functions (as in Session 1) - 30 minutes

2. An open-ended activity to build an interactive toy 2 hours

3. A brief activity to demonstrate the function of a motor 15 minutes

4. An open-ended activity to build an artefact using a motor 1 hour

Activities (1) and (3) were led by a researcher in collaboration with the class teacher, who demonstrated how to connect and program the components. During activities (2) and (4) groups worked independently, but each group was supported by an adult, who assisted the students with troubleshooting the TALKOO kit and debugging the visual programming. 


\subsection{Research questions, data sources and research variables}

The overarching research aim of this study was to identify human and machine observable differences in student behaviours in groups during CPS. This aim was shaped into three research questions:

- RQ1) What are the human observable differences between groups, in terms of the amount of time spent in different CPS competencies?

- RQ2) What are the machine observable differences between groups, in terms of nonverbal indexes of students physical interactivity?

- RQ3) What constructs of CPS can be identified using the nonverbal indexes of students physical interactivity?

Data sources and research variables for the first research question

The data source for the first research question takes the form of human collected observation data. The collection of this observation data was structured by a theoretical framework developed through previous empirical work: the PELARS CPS framework [11]. This framework was informed by the OECDs CPS assessment and encompasses three collaboration competencies (namely, establishing and maintaining shared understanding, taking appropriate actions to solve the problem, establishing and maintaining group organisation) and six problem-solving competencies (namely, identifying facts, representing and formulating knowledge, generating hypothesis, planning and executing, identifying knowledge and skill deficiencies, monitoring-reflecting-applying). The framework has been used to develop an observation protocol and mobile application that runs on phones, tablets and laptops.

During activities (2) and (4), each group was observed by an adult, who used the mobile application to code the instantiates of collaboration and problem solving, as defined by the protocol in the PELARS CPS framework. In order to ensure high level of agreement between different coders, all coders were trained in a day-long, hands-on workshop about the CPS competencies and the observation tool we built based on the framework. The human observers watched student activity and used the tool to mark the critical incidents that relate to the key dimensions for collaboration and problem-solving as they occurred. The tool recorded the exact date and time each dimension was marked by the human observer and we calculated the total amount of time spent on different dimensions of the CPS competencies.

The data collected with this observation tool was used to define two related research variables:

TPS $(\mathrm{G}, \mathrm{Ci})=$ Percentage of time each group $\mathrm{G}$ spent in each competence level Ci relative to problem-solving, where $\mathrm{G}=$ Red, Green, Purple, Blue, Yellow, Black and $\mathrm{i}=1,2,3,4,5,6$.

TCL $(\mathrm{G}, \mathrm{Ci})=$ Percentage of time each group $\mathrm{G}$ spent in each competence level $\mathrm{Ci}$ related to collaboration, where $\mathrm{G}=$ Red, Green, Purple, Blue, Yellow, Black and $\mathrm{i}=1,2,3$.

Data sources and research variables for the second research question 
In addition to the human observation data, we also collected video recordings of all of the empirical sessions. We analysed the video data using two variables that can also be automatically observed using our multimodal learning analytics system [12]:

a) students' hand positions, that can be used to represent their physical engagement with objects; b) students face directions, that might indicate their degree of involvement in the activity (depending on whether they are looking at the manipulated objects, at other students in their group, or at something outside the activity being carried out).

Video data analysis was performed by two researchers using a coding scheme, that is used to inform the future development of the automatic data capture facilities of our computer vision system. The coding scheme makes use of three digits, 0,1 and 2 to represent passive, semi-active and active student states. The active code (2) was used whenever a student's hand was active with an object; the semi-active code (1) was used when a student was not physically active, but their head was directed towards a peer who was active; and the passive code (0) was used for the rest of the situations. Students behaviours were coded using ten-second windows. To validate the coding, two coders applied this coding
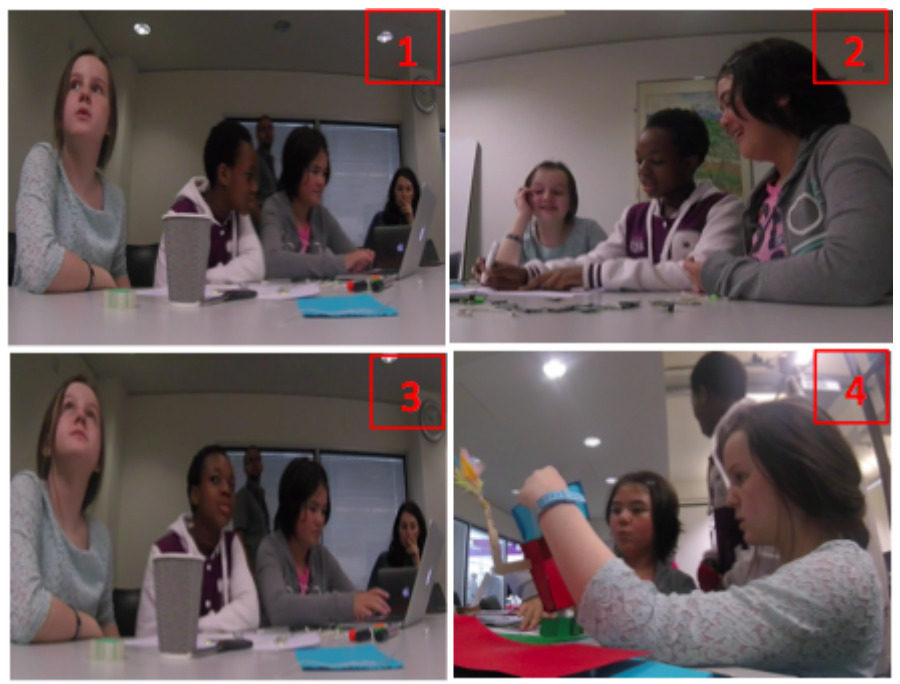

Fig. 1. Photos that show examples of situations coded as 012, 121, 002, 202

scheme to all groups video data using the 10- second window. This procedure was used as a way of testing the reliability of the coding system generated. Where there was disagreement, the researchers discussed the data and agreed a revised coding accordingly. Although, there was no objective measure for the inter-coder reliability, thanks to the simplicity of the coding scheme ( 0,1 ,and 2 codes), there were only a few disagreements between coders. 
To use this information in our research questions 2 we defined two research variables, designed to account for the physical activity for the group and for each individual student, respectively.

$\mathrm{N}(\mathrm{G}, \mathrm{i})=$ Percentage of $\mathrm{i}$ states in group $\mathrm{G}$, where $\mathrm{i}=0,1,2$ and $\mathrm{G}=\mathrm{Red}$, Green, Purple, Blue, Yellow, Black.

$\mathrm{Nj}(\mathrm{G}, \mathrm{i})=$ Percentage of $\mathrm{i}$ states for student $\mathrm{j}$ in group $\mathrm{G}$, where $\mathrm{j}=1,2,3 ; \mathrm{i}=$ $0,1,2$ and $\mathrm{G}=$ Red, Green, Purple, Blue, Yellow, Black.

Data sources and research variables for the third research question Finally, for the third research question we to represent the situation of each group at a given time by concatenating the activity code for each of its student at a given moment. For example, coding examples for the situations pictured in each of the four photographs are shown in Fig. 1.

The use of active, semi-active and passive codes provides 27 potential combinations for three students working together could be at any particular point in time. We categorized these positions into groups of 10 situations and identified potential predictors of CPS. Table 1 below presents this categorization (position and situations).

\begin{tabular}{|c|c|}
\hline $\begin{array}{l}\text { Potential positions of } \\
\text { CPS }\end{array}$ & $\begin{array}{l}\text { Categorised situations of three stu- } \\
\text { dents CPS }\end{array}$ \\
\hline 000 & Only 0s (000) \\
\hline $100,010,001$ & Two 0 , one 1 (001) \\
\hline $200,020,002$ & Two 0, one $2(002)$ \\
\hline $011,101,100$ & Two 1 , one $0(011)$ \\
\hline $012,021,102,120,201,210$ & One of each (012) \\
\hline 111 & Only 1s (111) \\
\hline $002,020,200$ & One 0 , two $2 \mathrm{~s}(022)$ \\
\hline $112,121,211$ & One two, two 1s (211) \\
\hline $122,212,221$ & One 1 , two 2s (221) \\
\hline 222 & Only 2s (222) \\
\hline
\end{tabular}

Table 1. Positions, situations and predictors

Next, we studied how we can use the learning sciences theories to make sense of students nonverbal indexes of physical interactions to create further signifiers of students CPS processes. To this end, we have investigated two concepts, namely 1) group synchrony and 2) individual accountability.

1) Group Synchrony: The quality of the collaboration is related with the quality of the relationships of the students within the groups [13]. This relationship quality is dependent on multiple aspects of group dynamics including reciprocity, impressions about others in the group, the feeling of being a community with other group members, and the perceptions about mutual dependency to achieve the aim [13]. Some of these psychosocial processes of social interactions might be interpreted through observation of students physical interactions. For instance, when groups are working well, students appear to converge their 
actions such that they move in unison [14]. In the learning analytics research context, Schneider and Pea [15] found that students visual synchrony, measured with eye-trackers, positively correlated with students learning gains. However, this finding was contradicted when it came to body synchrony. Schneider and Blikstein [16] found that even though gaze synchrony can be a strong predictor of student learning, body synchrony does not hold the same properties. In our study, we propose the use of a variable to account for synchrony in each group, which we define as $\operatorname{Syn}(G)=$ percentage of 222 states in group $G$, where $G=$ Red, ..., Black

2) Individual Accountability: Individual accountability refers to students making sure that they undertake their share of the work ad feel personally responsible for the groups success while others are also undertaking their share in completing the task. As argued by Slavin [17] in his synthesis of research so far undertaken in the domain group goals and individual accountability are the two key features of any successful group work. In groups that present high collaboration, students engage in promotive interaction and show a willingness to support each other in their joint efforts to complete the task and achieve the goal [17]. Therefore there appear to be two main requirements of individual accountability 1) students should undertake their share in completing the task, 2) each students share is promoted and acknowledged by other members of the group. In a learning analytics context, individual accountability is often considered to be measured with the amount of input generated by individual students. This satisfies the first requirement of individual accountability. However, individual students promotion and acknowledgement should also be taken into account in considerations of individual accountability. In order to interpret students promotion and acknowledgment of each others contribution, we added the percentage of those situations in which at least one member student is purposefully observing the action taken by a member of the group $(221+211)$ and subtracted those situations in which at least one student is ignoring an action taken by a member of the group $(220,210,200)$. That is, to represent individual accountability we have defined the following variable: $\mathrm{IA}(\mathrm{G})=$ percentage of 222 and 221 percentage of 220,210 and 200 , where $G=$ Red, ...., Black

\section{Independent variable: Classroom Teacher and Facilitators Judge- ment of Groups CPS}

In order to create an independent variable to categorise the differences between groups of students, the class teacher and facilitators involved in the activity were asked to use their expertise and experiences as teachers to judge each groups CPS competence. They were all asked to watch the video recordings of the six group sessions and to independently rank groups as high, medium and low competence CPS groups. Then, teachers and facilitators were brought together to discuss their individual judgments. In their individual judgments of the CPS competency of the groups, there were only discrepancies for two groups. Discussion between teachers and facilitators was used to agree a final competency value for these two groups. Table 2 below shows the results of this expert evaluation of groups CPS levels (see table 2 below). 
Table 2. Classification of students' groups according to their level of CPS

\begin{tabular}{rc}
\hline Colour Code of Group & Teachers' judgement of CPS competency \\
\hline Green & Low \\
Red & High \\
Purple & Medium \\
Blue & Medium \\
Yellow & High \\
Black & Low \\
\hline
\end{tabular}

\section{Results}

\subsection{Identifying Observable Differences in Terms of the Amount of Time Spent by Student Groups on Different CPS Competencies}

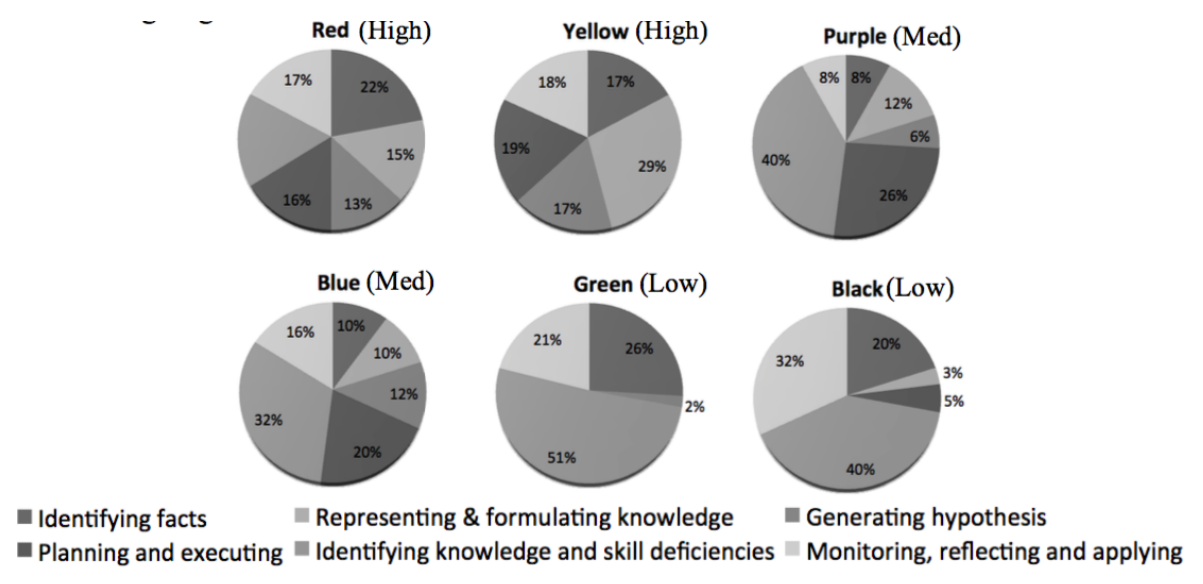

Fig. 2. Results for TPS (percentage of time each group devoted to each competency

As figures 2 and 3 present, the red and yellow groups (which were identified as high competency CPS groups by their teachers) show a more balanced segregation of different problem-solving activities: they spend their time fairly equally on the different dimensions of collaboration and problem-solving. By contrast, the other groups show unbalanced segregation of time spent in different CPS competencies. It appears that green and black groups (which were identified as low CPS groups by their teachers) spend most of their time on identifying knowledge and skill deficiencies. They spent very little or no time on the some of the important stages of problem solving, such as representing and formulating knowledge, generating hypotheses, and planning and executing. 
These behaviours might therefore be indicative of a less effective problem-solving pattern. The data from the red and yellow groups also evidences that they spent similar shares of time on different aspects of collaboration competencies. The green and purple groups, by comparison, present a greater difference in terms of the amount of time spent on the different aspects of collaboration. It appears that groups who had been evaluated as low CPS competent by human experts spent very little time on establishing and maintaining team organization, compared to other groups.

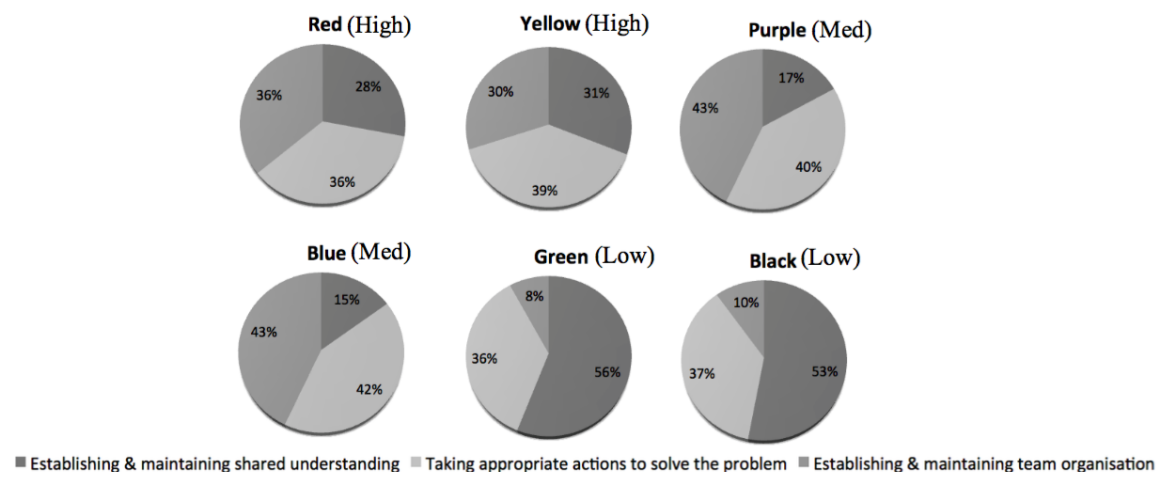

Fig. 3. Results for TCL (percentage of time each group devoted to each competency

The experts rated the red and yellow groups to be the high CPS competent groups, and it may, therefore, be the case that a balance between the different types of problem solving and collaboration activities is an indicator of effective CPS. Measuring the different amounts of time spent of key dimensions of CPS appears to be an effective method for identifying CPS competencies. However, it heavily relies on human observation of critical incidents. Next, we investigate machine observable features of CPS behaviours as part of our second and third research questions.

\subsection{Identifying Observable Differences in Nonverbal Indexes of Student Interactivity}

Figure 4 above illustrates that the percentage of active states (2) was similar across all six groups and ranged from $46.4 \%$ (Black) to $66.4 \%$ (Yellow). It is interesting to observe that the group with the highest percentage of active code (2), yellow group with $66.4 \%$, was judged as a high CPS group by human experts. Similarly, the group with the lowest of active codes (2), black group with $46.4 \%$, was rated by human experts as a low CPS competency group. However, this result does not lead to the conclusion that high active code percentage leads to high CPS competency. The other group rated by our experts as having low CPS competency (green group) had the second highest percentage of active codes (2), 


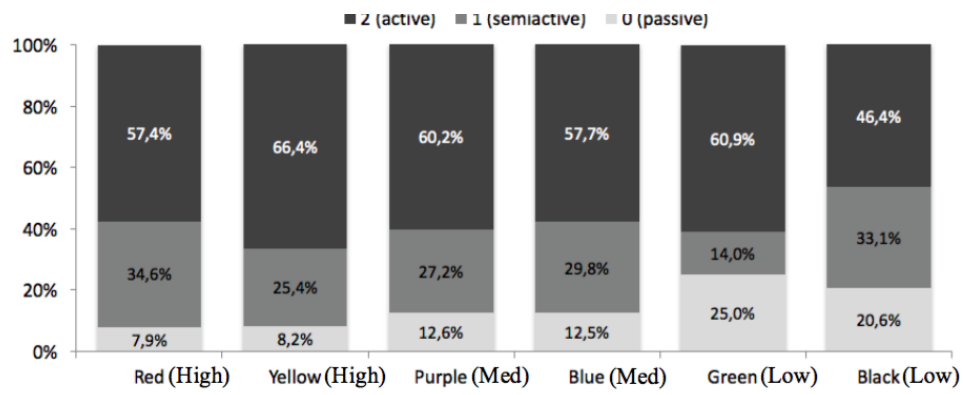

Fig. 4. Percentage of total number of passive 0 , semi-active 1 , and active 2 codes

and the other group rated as being high CPS competency (red group) has the second lowest percentage of active codes (2). This result suggests that the crude measure of the percentage of active states may not be a suitable indicator for differentiating the quality of the collaboration in the group (i.e. just individual students activity with objects may not be contributing to CPS overall). However, we also considered if students passive codes (0) might be a predictor. The red and yellow groups had the lowest percentages of passive codes (0). By contrast, the green and black groups had the highest percentages of passive codes (0). This result is surprising because the most researched and tracked indicators used in learning analytics research are often related to what students are doing. Our results suggest observing what students are not doing might be also informative.

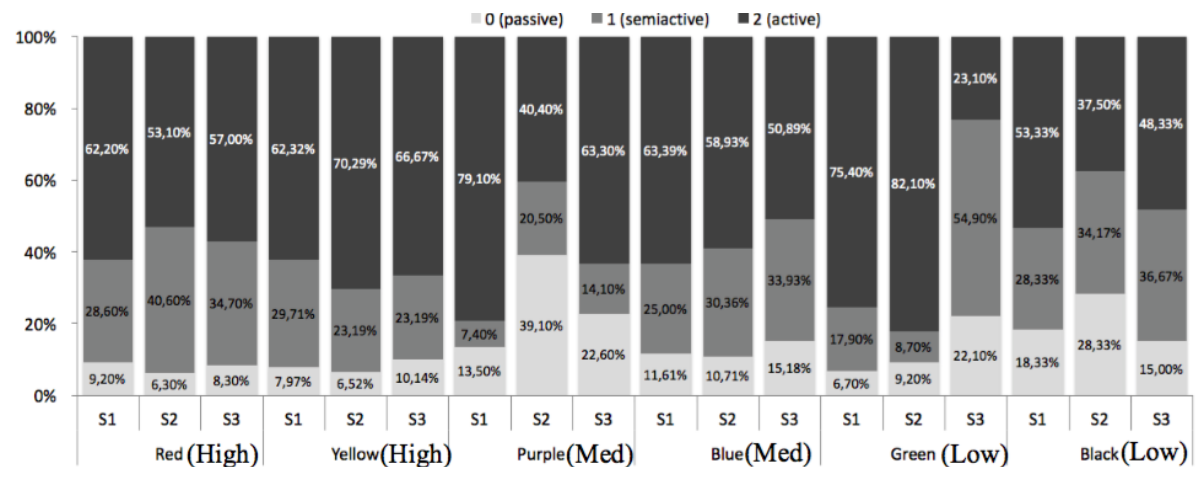

Fig. 5. Percentage of individual students number of passive 0 , semi-active 1 , and active 2 codes

We show in Fig. 5 the values obtained for the research variables $\mathrm{Nj}(\mathrm{G}, \mathrm{i})$. It illustrates that the individual students in the red and yellow groups get similar values for all the codes. The rest of the groups, by contrast, show greater differences between each students individual contributions. In the red group all three 
partners show a similar degree of involvement in the activity (2 code), ranging from $53,1 \%$ to $62,2 \%$. However, in the green group there is a greater difference in the degree of involvement (for S3 it is s $23.1 \%$ to and for $\mathrm{S} 2$ is $82,1 \%$ ). Clearly, in the red and yellow groups all members were contributing to the task similarly active ways, while in the other two groups students physical interactions were more passive and varied more./

\subsection{Identifying Potential Predictors of CPS}

Fig 6 presents the CPS rating of our human experts for each group and the percentages of different categories of situations. Note that the last two rows in this table correspond to the values of the proposed variables to measure the synchrony $\operatorname{Syn}(G)$ and individual accountability IA $(G)$, as defined previously.

\begin{tabular}{lcccccc} 
& Red & Yellow & Purple & Blue & Green & Black \\
\hline 000 & $2.40 \%$ & $0.50 \%$ & $7.4 \%$ & $6.4 \%$ & $3.6 \%$ & $4.2 \%$ \\
111 & $5.50 \%$ & $0.00 \%$ & $0.0 \%$ & $0.0 \%$ & $0.0 \%$ & $2.5 \%$ \\
001 & $0.90 \%$ & $1.00 \%$ & $0.0 \%$ & $0.0 \%$ & $0.9 \%$ & $2.5 \%$ \\
002 & $3.10 \%$ & $5.10 \%$ & $7.4 \%$ & $11.2 \%$ & $8.0 \%$ & $16.7 \%$ \\
011 & $0.90 \%$ & $1.00 \%$ & $0.0 \%$ & $0.0 \%$ & $0.0 \%$ & $1.7 \%$ \\
012 & $5.00 \%$ & $10.30 \%$ & $9.6 \%$ & $11.2 \%$ & $7.1 \%$ & $8.3 \%$ \\
022 & $2.80 \%$ & $12.80 \%$ & $18.8 \%$ & $8.0 \%$ & $27.7 \%$ & $25.8 \%$ \\
211 & $24.90 \%$ & $15.90 \%$ & $25.7 \%$ & $26.4 \%$ & $24.1 \%$ & $18.3 \%$ \\
221 & $30.10 \%$ & $36.40 \%$ & $20.1 \%$ & $24.8 \%$ & $23.2 \%$ & $11.7 \%$ \\
\hline SYN (G) & $24.50 \%$ & $16.90 \%$ & $11.0 \%$ & $12.0 \%$ & $5.4 \%$ & $8.3 \%$ \\
\hline IA (G) & $44.10 \%$ & $24.10 \%$ & $10.15 \%$ & $20.80 \%$ & $4.46 \%$ & $-20.83 \%$ \\
\hline Expert Evaluation & High & High & Medium & Medium & Low & Low \\
of CPS competency & & & & & &
\end{tabular}

Fig. 6. Percentages of the different situations across the six groups

The first feature that can be identified in Fig 6 is the total percentage of time that each group spent off-task (represented by the code 000) and the total percentage of time each group spent observing their teacher or a facilitator intervention (represented by the code 111). We can see that the percentage of off- task situations is very low, ranging from $0.4 \%$ in the yellow group to $7,4 \%$ in the purple one. Also, the low percentage of 111 states indicates little intervention from facilitators or teachers occurred. Perhaps more interestingly, the respective values of the two proposed measures, synchrony SYN $(G)$ and individual accountability IA $(G)$, show that groups rated by experts as being high CPS competent appear to have high percentages for both constructs. For instance, the red group spent $24.50 \%$ of their time in synchronized activity, whereas the green group only spent $5.4 \%$ of their time in synchronized activity. Similarly, for individual accountability, yellow groups calculated value is $24.10 \%$, whereas for the green group this value is $4.5 \%$. These results reveal an interesting correlation 
between the CPS quality of a group as judged by our human experts and their synchronization and individual accountability values calculated via nonverbal indexes of students physical interactivity. In the previously cited study [16], the researchers studied dyads collaborating remotely and found that body synchrony might not correlate with collaboration. However, the dynamics of three students working together in the same physical space on an open-ended task appear to be different.

\section{$5 \quad$ Discussion and Conclusions}

This paper reports an empirical study of young students engaging with CPS activities. We present several methods for identifying differences in groups CPS behaviour in PBL activities, based on human observation and students hand and head position data. We show that machine observable nonverbal indexes of student behaviours may be used to interpret certain educational constructs that are fundamental to CPS processes, such as individual accountability and synchronisation. The differences in group behaviours, presented by our data, provide evidence to support the suggestion that there might be a relationship between the competency of a groups CPS and their human and machine observable behaviours. This relationship requires further investigation, but our initial results are encouraging for those involved in the design of Learning Analytics. In this section, we discuss the answers to our research questions based on the three results sections presented above.

Our initial research question was to identify whether there are observable differences between group behaviours in terms of the amount of time spent on different CPS competencies. We used human observation data to answer this question and our results show clear differences between groups. Specifically, the high CPS competent groups spent more or less an equal distribution of time on their problem-solving stages. Whereas, the low CPS competent groups spent most of their time in identifying knowledge and skill deficiencies, whilst spending very little time or no time on other important aspects of problem-solving including, identifying facts, generating hypotheses, and representing knowledge. Although, appear to be effective, such human judgment based methods are hard to detect via learning analytics tools. However, such investigations of fine-grained actions of CPS can be used as tools to support the identification of knowledge distributions, to support the communication of knowledge inside groups, and, as a consequence of the cognitive group awareness, to facilitate organizational tasks. They can also be used to inform open learner models, to improve students reflective practice.

Our second research question required that we investigated the potential of students physical activity data, which is based on their hand and head positions. The results show that our coding scheme can provide useful data to identify group differences. First, these differences can be used to identify which students were left out or excluded from the CPS process. Second, and perhaps more importantly, the results show that, in high competent CPS groups, all students 
percentages of active, semi-active and passive scores overlapped and presented similar figures. However, in low CPS competent groups, individual students data did not illustrate similarities. In their early research Damon and Phelps [18] introduce two terms: equality and mutuality. Equality is a situation where participants are equal in status and participate in a two-way dialogue taking direction from one another; and mutuality is a situation where high mutuality means that discourse is extensive, intimate and connected. Authors argue that CPS should be high in both equality and mutuality. Looking at the results presented in figure 6 , some groups present more symmetrical individual contributions than others, which might reflect their effective CPS competencies in terms of their equality and mutuality.

Finally, our results show that students hand and head position data can be used to interpret group synchrony and individual accountability. Groups who were rated by human experts as having high CPS also presented a high percentage in group synchrony and individual accountability. We argue that the results of such differences, particularly, when they are triangulated with the data from other sources, may be used to identify effective CPS in an analytical and subjective way.

This exploratory study was limited to a small number of groups and, therefore, the results reported in this research paper should be approached with caution and we do not suggest that they are conclusive. However, we see the work presented here as an opportunity to lay the groundwork for future studies researching CPS in real-world environments under three research themes. First, our simple coding scheme of students active, semi-active and passive positions can inform the design of automated analysis systems of CPS from video data. Second, this study can inform the research in supervised machine learning approaches to automatically categorise students' CPS competences based on acquired multimodal data [19]. The observable features of CPS identified here can be used to label training data for algorithms. Third, human and machine observable features of CPS can be visualised with the purpose of improving reflective practice of students during their skill development activities.

Acknowledgements This work is co-funded by the European Union under the PELARS project. The fourth author was partially supported by Agencia Estatal de Investigación (AEI) y el Fondo Europeo de Desarrollo Regional (FEDER), TIN2016-80774-R.

\section{References}

1. Banks, F., \& Barlex, D. (2014). Teaching STEM in the secondary school: Helping teachers meet the challenge London: Routledge.

2. Kirschner, P. A., Sweller, J., \& Clark, R. E. (2006). Why minimal guidance during instruction does not work: An analysis of the failure of constructivist, discovery, problem-based, experiential, and inquiry-based teaching. Educational psychologist, 41(2), 75-86. 
3. Klahr, D., \& Nigam, M. (2004). The equivalence of learning paths in early science instruction effects of direct instruction and discovery learning. Psychological science, 15(10), 661-667.

4. Blikstein, P., \& Worsley, M. (2016). Multimodal learning analytics and education data mining: Using computational technologies to measure complex learning tasks. Journal of Learning Analytics, 3(2), 220-238.

5. Rodrguez, F. \& Boyer, K.E (2015). Discovering Individual and Collaborative Problem-Solving Modes with Hidden Markov Models. AIED 2015, pp. 408418. 6. Martnez-Maldonado, R; Kay, J. \& Yacef, K (2013). An Automatic Approach for Mining Patterns of Collaboration around an Interactive Tabletop, AIED 2013, pp. 101110.

7. OECD. (2015). Draft Collaborative Problem Solving Framework. Retrieved from http://www.oecd.org/pisa/pisaproducts/Draft PISA 2015 Collaborative Problem Solving Framework.pdf

8. Luckin, R., Baines, E., Cukurova, M., \& Holmes, W. (2017). Solved! Making the case for collaborative problem-solving. NESTA:UK.

9. Dillenbourg, P., \& Jermann, P. (2007). Designing Integrative Scripts. In F. Fischer, I. Kollar, H. Mandl, \& J. M. Haake (Eds.), Scripting Computer-Supported Collaborative Learning: Cognitive, Computational and Educational Perspectives (pp. 275-301). Boston, MA: Springer US.

10. Hmelo-Silver, C. E. (2004). Problem-based Learning: What and How do Students Learn. Educational Psychology Review, 16(3).

11. Cukurova, M., Avramides, K., Spikol, D., Luckin, R., \& Mavrikis, M. (2016). An analysis framework for collaborative problem solving in practice-based learning activities: a mixed-method approach, LAK16, pp. 84-88, ACM.

12. Ruffaldi, E., Dabisias, G., Landolfi, L., \& Spikol, D. (2016). Data collection and processing for a multimodal learning analytic system. SAI 2016, pp. 858-863. 13. Kreijns, K., Kirschner, P. A., \& Jochems, W. (2003). Identifying the pitfalls for social interaction in computer-supported collaborative learning environments: a review of the research. Computers in human behavior, 19(3), 335-353.

14. Lakens, D., \& Stel, M. (2011). If they move in sync, they must feel in sync. Social Cognition, 29(1)

15. Schneider, B., \& Pea, R. (2013). Real-time mutual gaze perception enhances collaborative learning and collaboration quality.International Journal of Computer-Supported Collaborative Learning, 8(4), 375-397.

16. Schneider, B., \& Blikstein, P. (2015). Unraveling students' interaction around a tangible interface using multimodal learning analytics. Journal of Educational Data Mining, 7(3).

17. Slavin, R. E. (1991). Synthesis of research of cooperative learning. Educational leadership, 48(5), 71-82.

18. Damon, W., \& Phelps, E. (1989). Critical distinctions among three approaches to peer education. International Journal of Educational Research, 13(1) 19. Spikol, D., Ruffaldi, E., \& Cukurova, M. (2017). Using Multimodal Learning Analytics to Identify Aspects of Collaboration in Project-Based Learning, CSCL17, Philadelphia, PA. 